\title{
Short communication: Antimicrobial susceptibility and frequency of resistance genes in Escherichia coli isolated from bovine mastitis
}

\author{
S. A. Metzger and J. S. Hogan ${ }^{1}$ \\ Department of Animal Sciences, The Ohio State University, Ohio Agricultural Research and Development Center, Wooster 44691
}

\begin{abstract}
Escherichia coli isolated from bovine milk samples submitted to the Ohio Agricultural Research and Development Center Mastitis Laboratory (Wooster) in 1985 to 1987 and in 2009 were compared for antimicrobial susceptibility and prevalence of antimicrobial resistance genes. Forty-four isolates from 1985 to 1987 and 55 isolates from 2009 were tested. Minimum inhibitory concentrations of 15 antimicrobials were determined using a commercially available broth microdilution system. Multiplex polymerase chain reaction was performed for gene detection. The percentage of isolates susceptible to trimethoprim/sulfamethoxazole, ampicillin, and kanamycin was lower in those collected in 1985 to 1987 than in isolates collected in 2009. Susceptibility did not differ between isolates from 1985 to 1987 and isolates from 2009 for the 12 other antimicrobials tested. A trimethoprim/sulfamethoxazole resistance gene was detected more frequently in isolates from 1985 to 1987 than in isolates from 2009. Gene frequencies for streptomycin resistance and tetracycline resistance were similar among 1985 to 1987 isolates and 2009 isolates. Resistance to most antimicrobials did not differ between isolates submitted to a mastitis diagnostic laboratory in 1985 to 1987 and those submitted in 2009. Changes observed indicated an increase in frequency of susceptibility in isolates to trimethoprim/ sulfamethoxazole, ampicillin, and kanamycin in 2009 isolates compared with 1985 to 1987 isolates.
\end{abstract}

Key words: mastitis, antimicrobial susceptibility, Escherichia coli

\section{Short Communication}

Antimicrobials generally have little effect on the clinical outcome of coliform mastitis because spontaneous cures often occur regardless of therapy (Hogan and Smith, 2003). In addition, many gram-negative bacteria are resistant to $\beta$-lactam drugs used in mastitis

Received November 21, 2012.

Accepted February 1, 2013.

${ }^{1}$ Corresponding author: hogan.4@osu.edu treatment (Watts et al., 1995; Salmon et al., 1998). The use of antimicrobials in agriculture has led to concerns about the potential for antimicrobial resistance to increase on farms and spread to human populations. However, Erskine et al. (2002) reported that susceptibility to several antimicrobials increased in many common mastitis pathogens from 1994 to 2000. Another study from 1994 to 2001 by Makovec and Ruegg (2003) reported similar trends in increasing susceptibility. Antimicrobial susceptibility of gram-negative mastitis pathogens did not change in South Korea from 2003 to 2008 (Nam et al., 2009). All 3 studies obtained bacteria from mastitic milk samples that had been submitted to a diagnostic laboratory for testing. The National Mastitis Council (Verona, WI) conducted a review of antimicrobial resistance studies and concluded that no evidence existed of increasing antimicrobial resistance among mastitis pathogens (Erskine et al., 2004). The purpose of the current trial was to compare phenotypic antimicrobial susceptibility and prevalence of antimicrobial resistance genes in Escherichia coli isolated from milk samples submitted to a mastitis diagnostic laboratory in 1985 to 1987 and 2009.

Escherichia coli $(\mathrm{n}=99)$ isolates tested were from aseptically collected quarter-milk samples submitted to the Ohio Agricultural Research and Development Center Mastitis Laboratory (Wooster). Samples were submitted from 1985 to $1987(\mathrm{n}=44)$ or in $2009(\mathrm{n}=$ 55). Primary culture for all quarter-milk samples was surface plating $10 \mu \mathrm{L}$ of milk on Trypticase soy agar (Becton, Dickinson and Co., Sparks, MD) with 5\% bovine blood and $0.1 \%$ esculin and $100 \mu \mathrm{L}$ of milk on MacConkey agar (Becton, Dickinson and Co.). Primary cultures were incubated aerobically at $37^{\circ} \mathrm{C}$ and plates were examined at 24 and $48 \mathrm{~h}$. Escherichia coli were presumptively identified by colony morphology, cellular morphology, Gram reaction, motility, triple sugar iron, and citrate utilization tests (Hogan et al., 1999). Escherichia coli were identified biochemically using the API 20E system (bioMérieux, Marcy-l'Étoile, France). Escherichia coli ATCC 25922 was used as a positive control isolate in biochemical testing. Confirmed isolates were stored in Trypticase soy broth plus $20 \%$ glycerin at $-80^{\circ} \mathrm{C}$ until testing. Isolates collected from 1985 to 
Table 1. Primer sets used for detection of antimicrobial genes from Escherichia coli isolated from milk samples submitted to a mastitis diagnostic laboratory from 1985 to 1987 and 2009

\begin{tabular}{|c|c|c|c|c|c|}
\hline \multirow[b]{2}{*}{ Primer set } & \multirow[b]{2}{*}{ Gene } & \multicolumn{2}{|r|}{ Primer } & \multirow{2}{*}{$\begin{array}{c}\text { Annealing } \\
\text { temperature }\left({ }^{\circ} \mathrm{C}\right)\end{array}$} & \multirow{2}{*}{$\begin{array}{c}\text { Fragmen } \\
\text { size (bp) }\end{array}$} \\
\hline & & Direction & Sequence & & \\
\hline \multirow[t]{5}{*}{1} & $\operatorname{aad} A$ & $\begin{array}{l}\text { Forward } \\
\text { Reverse }\end{array}$ & $\begin{array}{l}\text { GTGGATGGCGGCCTGAAGCC } \\
\text { AATGCCCAGTCGGCAGCG }\end{array}$ & 68 & 525 \\
\hline & sulI & Forward & GTGACGGTGTTCGGCATTCT & & \\
\hline & & Reverse & CCTGGTGATAACGGCAATTC & 68 & 779 \\
\hline & str $A$ & Forward & CCTGGTGATAACGGCAATTC & & \\
\hline & & Reverse & CCAATCGATAACGGCAATTC & 55 & 546 \\
\hline \multirow[t]{4}{*}{2} & $\operatorname{str} B$ & Forward & ATCGTCAAGGGATTGAAACC & 55 & 509 \\
\hline & & Reverse & GGATCGTAGAACATATTGGC & & \\
\hline & tet $A$ & Forward & GGATCGTAGAACATATTGGC & 64 & 502 \\
\hline & & Reverse & CGGCAGGCAGAGCAAGTAGA & 64 & 502 \\
\hline \multirow[t]{4}{*}{3} & tet $B$ & Forward & CATTAATAGGCGCATCGCTG & & \\
\hline & & Reverse & TGAAGGTCATCGATAGCAGG & 64 & 930 \\
\hline & tet $C$ & Forward & GCTGTAGGCATAGGCTTGGT & 64 & 888 \\
\hline & & Reverse & GCCGGAAGCGAGAAGAATCA & 04 & 000 \\
\hline
\end{tabular}

1987 were tested every 36 to 48 mo for ability to grow aerobically on MacConkey agar until the current trial was initiated in September, 2009.

Minimum inhibitory concentrations of antimicrobials were determined by microdilutions in commercially available plates (Sensititre gram-negative NARMS; Trek Diagnostic Systems Inc., Cleveland, OH). Microdilution plates included amikacin, ampicillin, amoxicillin/clavulanic acid, ceftriaxone, chloramphenicol, cefoxitin, trimethoprim/sulfamethoxazole, gentamicin, kanamycin, nalidixic acid, sulfisoxazole, streptomycin, tetracycline, and ceftiofur. Isolates were inoculated on esculin blood agar and incubated at $37^{\circ} \mathrm{C}$ for $24 \mathrm{~h}$. After incubation, several colonies of each isolate were emulsified in distilled water and diluted in Mueller-Hinton broth (Trek Diagnostic Systems Inc.) to approximately $1 \times 10^{5} \mathrm{cfu} / \mathrm{mL}$. Microdilution plates were inoculated with the bacterial culture, covered with an adhesive seal, and incubated at $37^{\circ} \mathrm{C}$ for $24 \mathrm{~h}$. After $24 \mathrm{~h}$ of incubation, plates were read with a manual plate reader (Sensititre Manual Viewer; Trek Diagnostic Systems Inc.). Isolates were classified as susceptible, intermediate, or resistant according to National Committee on Clinical Laboratory Standards (NCCLS) standards (CLSI, 2008). Escherichia coli ATCC 25922 was used as a control.

Deoxyribonucleic acid was purified from E. coli (Generation Capture Column; Qiagen Inc., Valencia, $\mathrm{CA}$ ) and antimicrobial resistance genes were amplified (Qiagen Multiplex PCR Kit; Qiagen Inc.) in a thermal cycler (Hybrid Sprint; Thermo Electron Corp., Milford, MA.) The initial activation step was $15 \mathrm{~min}$ at $95^{\circ} \mathrm{C}$; DNA was then denatured at $94^{\circ} \mathrm{C}$ for $30 \mathrm{~s}$, annealed for $90 \mathrm{~s}$ at the given annealing temperature for each primer set (Lanz et al., 2003; Table 1), and extended at $72^{\circ} \mathrm{C}$ for $90 \mathrm{~s}$. This cycle was performed 34 times before the final extension step at $72^{\circ} \mathrm{C}$ for $15 \mathrm{~min}$. The PCR products were stored at $-28^{\circ} \mathrm{C}$. Samples were loaded (GelPilot DNA Loading Dye; Qiagen Inc.), electrophoresed in 2.0\% agarose (NuSieve GTG Agarose; Lonza Rockland Inc., Rockland, ME) gels at $100 \mathrm{~V}$ and 200 $\mathrm{mA}$ for $2 \mathrm{~h}$, and photographed (Gel Doc XR; Bio-Rad Laboratories Inc., Hercules, CA).

The proportion of isolates that were susceptible to each antimicrobial was calculated for both time periods. The proportion of isolates with each gene combination was calculated for both time periods. Differences in frequencies of antimicrobial susceptibility or genes were compared using a Fisher exact test when an outcome had fewer than 5 results. A $\chi^{2}$ test was used when an outcome had more than 5 results (Sokal and Rohlf, 1981). Results were considered significant at $P<0.05$.

Distributions of MIC were similar between $E$. coli isolated from milk samples submitted from 1985 to 1987 (Table 2) and those from 2009 (Table 3) for most antimicrobials. Changes observed indicated an increase $(P<0.05)$ in frequency of susceptibility in isolates to ampicillin, trimethoprim/sulfamethoxazole, and kanamycin (Table 4) in those from 2009 compared with isolates from 1985 to 1987 . The percentage of isolates susceptible did not decrease in isolates from 2009 compared with isolates from 1985 to 1987 for any of the antimicrobials tested. The percentage of isolates susceptible to tetracycline, streptomycin, chloramphenicol, nalidixic acid, amoxicillin/clavulanic acid, sulfisoxazole, gentamicin, cefoxitin, and ceftiofur did not differ between isolates from 1985 to 1987 and isolates from 2009. All 99 Escherichia coli isolates from both 1985 to 1987 and 2009 were susceptible to amikacin, ceftriaxone, and ciprofloxacin.

The percentage of isolates susceptible to ampicillin increased from $79.5 \%$ of isolates in 1985 to 1987 to 
ఠ Ampicilli

$\delta \quad$ Cefoxitin

\& Ceftriaxone

z Chloramphenicol

or Gentamicin

N Kanamycin

$\stackrel{\circ}{\vec{\omega}}$ Nalidixic acid

Streptomycin

Sulfisoxazole

Tetracycline

\begin{tabular}{|c|c|c|c|c|c|c|c|c|c|c|}
\hline 0.15 & 0.03 & 0.12 & 0.25 & 0.5 & 1 & 2 & & 4 & & 8 \\
\hline \multirow{8}{*}{41} & \multirow{8}{*}{3} & & \multirow{8}{*}{$\begin{array}{l}10 \\
43\end{array}$} & & 1 & 23 & 18 & & 2 & \\
\hline & & & & & 3 & 17 & 14 & & 1 & \\
\hline & & & & & & 4 & 30 & & 10 & \\
\hline & & & & 33 & 1 & & & $\dagger$ & & $\ddagger$ \\
\hline & & & & 1 & & & & & & \\
\hline & & & & & & $\dagger$ & \multirow{2}{*}{9} & $\ddagger$ & & $\ddagger$ \\
\hline & & & & 21 & 21 & 1 & & & & $\dagger$ \\
\hline & & & & & 2 & 22 & 19 & & $\begin{array}{r}33 \\
1\end{array}$ & \\
\hline
\end{tabular}

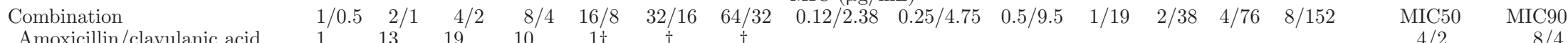

31

$\mathrm{MIC}(\mu \mathrm{g} / \mathrm{mL})$

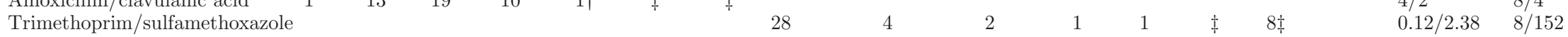

${ }^{1}$ MIC50 $=$ MIC required to inhibit the growth of 50\% of organisms; MIC90 = MIC required to inhibit the growth of $90 \%$ of organisms.

$\dagger$ Intermediate values; łresistant values.

Table 3. Distribution of MIC of selected antimicrobials for Escherichia coli isolated from milk samples submitted to a mastitis diagnostic laboratory in 2009 (n $=55)^{1}$

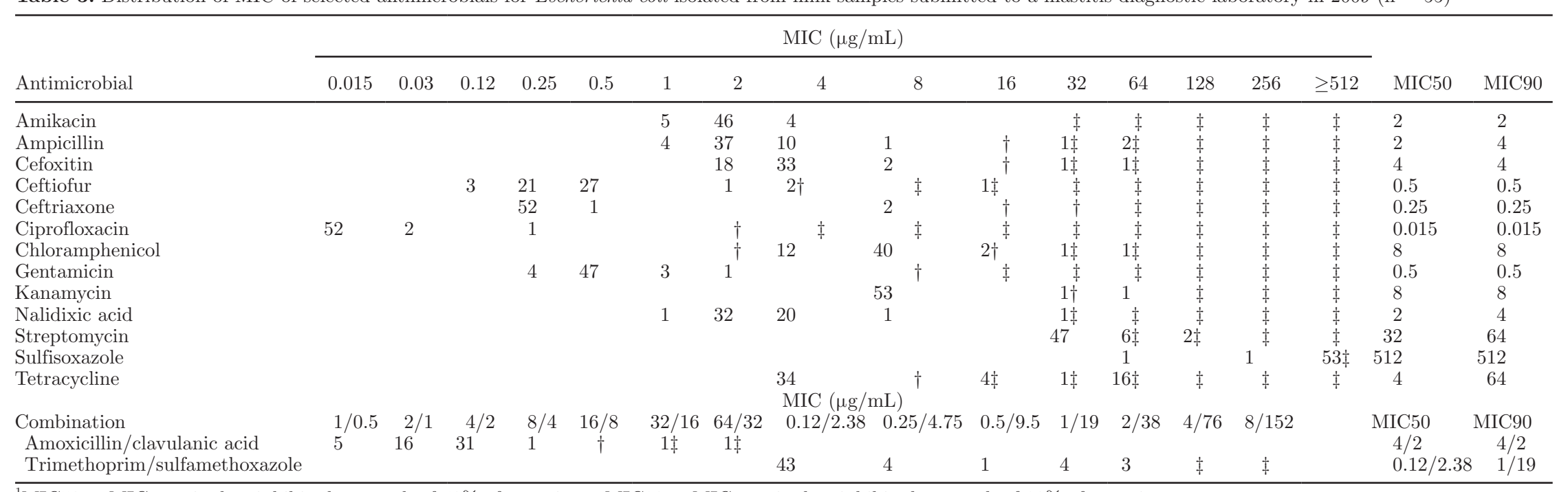

${ }^{1}$ MIC50 $=$ MIC required to inhibit the growth of 50\% of organisms; MIC90 = MIC required to inhibit the growth of $90 \%$ of organisms.

$\dagger$ Intermediate values; łresistant values. 
Table 4. Percentage distribution of susceptible Escherichia coli isolated from milk samples submitted to a mastitis diagnostic laboratory from 1985 to $1987(\mathrm{n}=44)$ and in $2009(\mathrm{n}=55)$

\begin{tabular}{lccc}
\hline & \multicolumn{2}{c}{ Year (\%) } & \\
\cline { 2 - 3 } Antimicrobial & $1985-1987$ & 2009 & $P$-value \\
\hline Amikacin & 100.0 & 100.0 & 1.0 \\
Ampicillin & 79.5 & 94.5 & 0.031 \\
Cefoxitin & 100.0 & 96.4 & 0.50 \\
Ceftiofur & 100.0 & 94.5 & 0.25 \\
Ceftriaxone & 100.0 & 100.0 & 1.0 \\
Ciprofloxacin & 100.0 & 100.0 & 1.0 \\
Chloramphenicol & 93.2 & 89.1 & 1.0 \\
Gentamicin & 97.7 & 100.0 & 0.44 \\
Kanamycin & 77.3 & 96.4 & 0.002 \\
Nalidixic acid & 97.7 & 100.0 & 0.44 \\
Streptomycin & 75.0 & 85.5 & 0.21 \\
Sulfisoxazole & 6.8 & 3.6 & 0.65 \\
Tetracycline & 70.5 & 61.8 & 0.40 \\
Amoxicillin/clavulanic acid & 97.7 & 96.4 & 1.0 \\
Trimethoprim/sulfamethoxazole & 81.8 & 100.0 & 0.001 \\
\hline
\end{tabular}

94.5\% of isolates in 2009. A similar trend in susceptibility was reported in E. coli from 1994 to 2000, with susceptibility increasing from $78.2 \%$ of isolates to $85.5 \%$ of isolates (Erskine et al., 2002). Ampicillin susceptibility remained steady at $78 \%$ of isolates in a 1994 to 2001 study (Makovec and Ruegg, 2003). Ampicillin susceptibility in South Korean E. coli isolates from 2003 to 2008 did not change, and ranged from 60.0 to $72.9 \%$ (Nam et al., 2009). In the United States, non-cephalosporin $\beta$-lactams such as ampicillin comprised $33.8 \%$ of antimicrobials used in mastitis treatments in 2002 (USDAAPHIS, 2008). The use of non-cephalosporin $\beta$-lactams decreased to $19.1 \%$ of mastitis antimicrobials in 2007 , suggesting a possible relationship with the susceptibility increase found in the current study (USDA-APHIS, 2008).

Resistance to sulfonamides has been observed in $E$. coli from animal sources since 1964, as these are some of the most commonly used drugs in animal production systems in the last $70 \mathrm{yr}$ (Tadesse et al., 2012). However, sulfonamides such as trimethoprim/sulfamethoxazole were only used in $1.2 \%$ of cows treated for mastitis in the United States in 2007 (USDA-APHIS, 2008). Trimethoprim/sulfamethoxazole susceptibility increased from $81.8 \%$ of isolates in 1985 to 1987 to $100.0 \%$ of isolates in $2009(P<0.001)$. Susceptibility to trimethoprim/sulfamethoxazole did not change in a study of mastitis-causing E. coli from 1994 to 2001 (Makovec and Ruegg, 2003) nor in a study of mastitis-causing E. coli from 1994 to 2000 (Erskine et al., 2002). Both Erskine et al. (2002) and Makovec and Ruegg (2003) found trimethoprim/sulfamethoxazole susceptibility to be greater than $94 \%$. A South Korean study (Nam et al., 2009) found trimethoprim susceptibilities between 80 and $90 \%$ from 2003 to 2008. Lehtolainen et al. (2003) found that $96 \%$ of mastitis-causing $E$. coli isolates from Israeli dairy farms and $98 \%$ of isolates from Finnish dairies were resistant to trimethoprim/sulfadiazine, indicating that susceptibility to sulfonamide drugs remains high among mastitis-causing E. coli worldwide. In the current trial, 8 E. coli isolates from 1985 to 1987 were resistant to trimethoprim/sulfamethoxazole, and 7 of these resistant isolates (87.5\%) were positive for sulI. The frequency of sulI decreased from $15.9 \%$ of isolates in 1985 to 1987 to $0 \%$ of isolates in $2009(P<0.01)$. In a Swiss study, $53 \%$ of sulfonamide-resistant $E$. coli isolates from various animal sources were positive for the sulI gene (Lanz et al., 2003). All 2009 E. coli isolates were susceptible to trimethoprim/sulfamethoxazole. All susceptible isolates from both 1985 to 1987 and 2009 were negative for sulI.

Kanamycin susceptibility increased from $77.3 \%$ of 1985 to 1987 isolates to $96.4 \%$ of 2009 isolates $(P<$ 0.01), contrasting with results of a South Korean study in which kanamycin susceptibility varied widely from year to year but did not change overall from 2003 to 2008 (Nam et al., 2009). Most studies testing antimicrobial susceptibility did not test for kanamycin susceptibility, possibly because aminoglycosides such as kanamycin are generally not used in mastitis treatment in the United States (USDA-APHIS, 2008).

Tetracyclines only comprised $2.0 \%$ of mastitis treatments in the United States in 2007, but were commonly used in medicated milk replacer for preweaned heifers (Sawant et al., 2005; Raymond et al., 2006; USDA-APHIS, 2008). Susceptibility to tetracycline did not differ between 1985 to 1987 isolates and 2009 isolates in the current study. Susceptibility was $70.5 \%$ among older isolates and $61.8 \%$ among more recent isolates. These results are similar to 2 previous stud- 
ies of mastitis pathogens in the United States (Erskine et al., 2002; Makovec and Ruegg, 2003). Tetracycline resistance genes such as tet $A, \operatorname{tet} B$, and tet $C$ are often found on plasmids and could potentially be spread from calf environments to cow environments (Travis et al., 2006; Bradford and Dean, 2007). Although a tendency toward a higher frequency of tetracycline resistance determinants were in resistant E. coli isolates in 2009 compared with those from 1985 to 1987, the prevalence of these 3 genes in the current study did not differ between isolates from the 2 time periods. In addition the frequency of tetracycline resistance determinants reflected phenotypic antimicrobial susceptibility. The tet gene frequencies did not differ between time periods for tetracycline-resistant isolates or for all isolates. Tetracycline resistance determinants were found in 61.5 and $90.5 \%$ of tetracycline resistance $E$. coli isolates from 1985 to 1987 and 2009, respectively. The tet $A$ gene was not present in any 1985 to 1987 isolates. Only one isolate, from 2009, was positive for 2 tetracycline resistance determinants (tet $A$ and tet $B$ ). The tet $A$ gene alone was found in $0 \%$ of 1985 to 1987 isolates and $19.0 \%$ of 2009 isolates. The tet $B$ gene alone was found in $7.7 \%$ of 1985 to 1987 isolates and $14.3 \%$ of 2009 isolates. The tetC gene alone was found in $53.8 \%$ of 1985 to 1987 isolates and $52.4 \%$ of 2009 isolates. Isolates that were not susceptible to tetracycline generally were positive for at least one of the tet genes tested.

Streptomycin was a component in intramammary antimicrobials administered at dry-off to $36.9 \%$ of cows receiving dry cow therapy in the United States (USDA, 2009). Streptomycin susceptibility did not differ between time periods in the current study or in a South Korean study of bovine mastitis-causing E. coli (Nam et al., 2009). Streptomycin resistance gene frequency among all isolates did not differ between time periods in the current trial. Isolates that were not susceptible to streptomycin in the current study were generally positive for at least one streptomycin resistance gene. The $\operatorname{str} A$ and $\operatorname{str} B$ genes were carried together on a single plasmid in a study of porcine $E$. coli, whereas aadA was found on a separate plasmid (Travis et al., 2006). The $\operatorname{str} B$ gene was always found with $\operatorname{str} A$ in the current study and in all but 1 of 101 strB-positive isolates in a Swiss study (Lanz et al., 2003). In both the Swiss study and the current study, the most common gene combination was for isolates to be negative for aadA and positive for both $\operatorname{str} A$ and $\operatorname{str} B$ (Lanz et al., 2003). A total of $63.6 \%$ of streptomycin-resistant isolates from 1985 to 1987 and $87.5 \%$ from 2009 were positive for both $\operatorname{str} A$ and $\operatorname{str} B$ but negative for $a a d A$. For streptomycinresistant isolates from 1985 to $1987,9.1 \%$ did not have aadA, str $A$, or $\operatorname{str} B$ whereas $12.5 \%$ of 2009 isolates were negative for all 3 tested streptomycin-resistance deter- minants. None of the streptomycin-resistant 2009 isolates and $9.1 \%$ of 1985 to 1987 streptomycin-resistant isolates were positive for aadA and negative for both $\operatorname{str} A$ and $\operatorname{str} B$. All 3 resistance determinants were found together in $18.2 \%$ of 1985 to 1987 isolates, but aadA was not detected in any 2009 isolates. Both strA and strB were detected in three 2009 isolates that were not resistant to streptomycin.

Antimicrobial resistance in E. coli has increased in isolates from human populations and various foodproducing animals (Tadesse, et al., 2012), but does not appear to be changing in the same manner in bovine mastitis-causing E. coli. For example, antimicrobial susceptibility trends in $E$. coli isolates from bovine milk samples differed from antimicrobial susceptibility trends in E. coli isolates from human urinary tract infections or bloodstream infections. Susceptibilities to ampicillin and trimethoprim/sulfamethoxazole increased among mastitis isolates from 2009 and 1985 to 1987. Isolates from urinary tract infections in women had lower susceptibilities to ampicillin, with only 60 to $70 \%$ of isolates susceptible (Gupta et al., 2001; Karlowsky et al., 2002; Olson et al., 2009). Trimethoprim/sulfamethoxazole susceptibility of urinary tract infection isolates was similar to 1985 to 1987 mastitis isolates but lower than the susceptibility of 2009 isolates. A study of E. coli isolated from human bloodstream infections measured decreases in susceptibility to ampicillin, trimethoprim/ sulfamethoxazole, and ciprofloxacin from 1998 to 2007 (Al-Hasan et al., 2009). Ampicillin susceptibility among bloodstream isolates was initially $68 \%$, similar to urinary tract infection isolates and mastitis isolates from 1985 to 1987, but decreased to $48 \%$ in 2007 (Al-Hasan et al., 2009). Ciprofloxacin susceptibility also decreased from $100 \%$ of 1998 bloodstream isolates to $88 \%$ of 2007 bloodstream isolates (Al-Hasan et al., 2009). Escherichia coli isolated from urinary tract infections and bloodstream infections in humans appear to exhibit different changes in susceptibility than $E$. coli isolated from bovine milk samples.

\section{REFERENCES}

Al-Hasan, M. N., B. D. Lahr, J. E. Eckel-Passow, and L. M. Baddour. 2009. Antimicrobial resistance trends of Escherichia coli bloodstream isolates: A population-based study, 1998-2007. J. Antimicrob. Chemother. 64:169-174.

Bradford, P. A., and C. R. Dean. 2007. Resistance of gram-negative bacilli to antimicrobials. Pages 97-159 in Antimicrobial Resistance and Implications for the Twenty-First Century. I. W. Fong and K. Drlica, ed. Springer, New York, NY.

CLSI (Clinical and Laboratory Standards Institute). 2008. Performance standards for antimicrobial disk and dilution susceptibility tests for bacteria isolated from animals; approved standard-Third edition. CLSI document M31-A3. CLSI, Wayne, PA.

Erskine, R., J. Cullor, M. Schaellibaum, B. Yancey, and A. Zecconi. 2004. Bovine mastitis pathogens and trends in resistance to an- 
tibacterial drugs. Mastitis Council Research Committee Report. National Mastitis Council (NMC) Annual Meeting Proceedings. NMC, Verona, WI.

Erskine, R. J., R. D. Walker, C. A. Bolin, P. C. Bartlett, and D. G. White. 2002. Trends in antibacterial susceptibility of mastitis pathogens during a seven-year period. J. Dairy Sci. 85:1111-1118.

Gupta, K., D. F. Sahm, D. Mayfield, and W. E. Stamm. 2001. Antimicrobial resistance among uropathogens that cause communityacquired urinary tract infections in women: A nationwide analysis. Clin. Infect. Dis. 33:89-94.

Hogan, J. S. R. N. González, R. J. Harmon, S. C. Nickerson, S. P. Oliver, J. W. Pankey, and K. L. Smith. 1999. Laboratory and Field Handbook on Bovine Mastitis. Natl. Mastitis Counc. Inc., Verona, WI

Hogan, J., and K. L. Smith. 2003. Coliform mastitis. Vet. Res. 34:507-519.

Karlowsky, J. A., L. J. Kelly, C. Thornsberry, M. E. Jones, and D. F. Sahm. 2002. Trends in antimicrobial resistance among urinary tract infection isolates of Escherichia coli from female outpatients in the United States. Antimicrob. Agents Chemother. 46:25402545 .

Lanz, R., P. Kuhnert, and P. Boerlin. 2003. Antimicrobial resistance and resistance genes determinants in clinical Escherichia coli from different animal species in Switzerland. Vet. Microbiol. 91:73-84.

Lehtolainen, T., A. Shwimmer, N. Y. Shpigel, T. Honkanen-Buzalski, and S. Pyörälä. 2003. In vitro antimicrobial susceptibility of Escherichia coli isolates from clinical bovine mastitis in Finland and Israel. J. Dairy Sci. 86:3927-3932.

Makovec, J. A., and P. L. Ruegg. 2003. Antimicrobial resistance of bacteria isolated from dairy cow milk samples submitted for bacterial culture: 8,905 samples (1994-2001). J. Am. Vet. Med. Assoc. $222: 1582-1589$

Nam, H. M., S. K. Lim, H. M. Kang, J. M. Kim, J. S. Moon, K. C. Jang, J. M. Kim, Y. S. Joo, and S. C. Jung. 2009. Prevalence and antimicrobial susceptibility of gram-negative bacteria isolated from bovine mastitis between 2003 and 2008 in Korea. J. Dairy Sci. $92: 2020-2026$.

Olson, R. P., L. J. Harrell, and K. S. Kave. 2009. Antibiotic resistance in urinary isolates of Escherichia coli from college women with urinary tract infections. Antimicrob. Agents Chemother. $53: 1285-1286$

Raymond, M. J., R. D. Wohrle, and D. R. Call. 2006. Assessment and promotion of judicious antibiotic use on dairy farms in Washington state. J. Dairy Sci. 89:3228-3240.

Salmon, S. A., J. L. Watts, F. M. Aarestrup, J. W. Pankey, and R. J. Yancey Jr. 1998. Minimum inhibitory concentrations for selected antimicrobial agents against organisms isolated from the mammary glands of dairy heifers in New Zealand and Denmark. J. Dairy Sci. 81:570-578

Sawant, A. A., L. M. Sordillo, and B. M. Jayarao. 2005. A survey on antibiotic usage in dairy herds in Pennsylvania. J. Dairy Sci. 88:2991-2999.

Sokal, R. R., and F. J. Rohlf. 1981. Analysis of frequencies. Pages 738-747 in Biometry. 2nd ed. W. H. Freeman and Co., San Francisco, CA.

Tadesse, D. A., S. Zhao, E. Tong, S. Ayers, A. Singh, M. J. Bartholomew, and P. F. McDermott. 2012. Antimicrobial drug resistance in Escherichia coli from humans and food animals, United states, 1950-2002. Emerg. Infect. Dis. 18:741-749.

Travis, R. M., C. L. Gyles, R. Reid-Smith, C. Poppe, S. A. McEwen, R. Friendship, N. Janecko, and P. Boerlin. 2006. Chloramphenicol and kanamycin resistance among porcine Escherichia coli in Ontario. J. Antimicrob. Chemother. 58:173-177.

USDA (US Department of Agriculture). 2009. Dairy 2007, Part V: Changes in Dairy Cattle Health and Management Practices in the United States, 1996-2007. USDA:Animal and Plant Health Inspection Service:Veterinary Services (APHIS:VS), Centers for Epidemiology and Animal Health (CEAH), Fort Collins, CO.

USDA-APHIS (US Department of Agriculture-Animal and Plant Health Inspection Service). 2008. Antibiotic use on U. S. dairy operations, 2002 and 2007. USDA N534.1008. USDA, Fort Collins, CO.

Watts, J. L., S. A. Salmon, R. J. Yancey Jr., S. C. Nickerson, L. J. Weaver, C. Holmberg, J. W. Pankey, and L. K. Fox. 1995. Antimicrobial susceptibility of microorganisms isolated from the mammary glands of dairy heifers. J. Dairy Sci. 78:1637-1648. 